\title{
Object Tracking Algorithm Based on Camshift and Grey Prediction Model in Occlusions
}

\author{
Zhiwei Wang \\ College of Computer Science and Information \\ Guizhou University \\ Guizhou, China,550025 \\ wangzhiwei06@163.com
}

\author{
Fuping Yang \\ College of Computer Science and Technology \\ Chongqing University of Posts and \\ Telecommunications \\ Chongqing, China ,400065 \\ yangfp@cqupt.edu.cn
}

\begin{abstract}
In this paper, an improved object tracking algorithm based on Camshift and grey prediction model GM (1.1) was proposed to solve problem of the poor tracking ability in occlusions. The whole tracking system could be divided into three parts: the occlusions identifying system based on Bhattacharyya coefficient analysis, the prediction system based on grey model GM (1.1) and Camshift algorithm. In the tracking process, those three parts run by turns according to different cases. Experiment results of variant video sequences demonstrate that the proposed method can track the objects stably and accurately during occlusions.
\end{abstract}

Keywords-Occlusions, Grey prediction, Camshift, Object tracking

\section{INTRODUCTION}

In recent years, object tracking has become one of the most active research areas in computer vision field, and a lot of different object tracking algorithms and their related applications have been proposed. Mean shift algorithm with quickly matching mode is widely used in object tracking on the basis of the estimation theory of nonparametric kernel probability density [1]. In 1975, the mean shift algorithm was first proposed by Fukunaga et al [2] and applied to pattern recognition. In 1995, Cheng [3] introduced it to the field of computer vision. In 1999, the mean shift algorithm was applied by Comaniciu [4] to object tracking. But the mean shift algorithm is not able to update the object model in the process of tracking, which will result in inaccurate scale locating and even object losing while object's scale varies obviously. Camshift (continuous adaptive of mean shift) algorithm is proposed by Bradski [5] in order to solve such problems. This algorithm can automatically adjust the window size to fit the size of object changes in the image. It is effective to resolve the problem of inaccurate object tracking due to the deformation of moving object. In [6], Sum and Liu solve the object losing problem due to the acceleration of object by using Camshift algorithm and adaptive expanding the search window. However, the track process of Camshift algorithm is easily affected by the object of similar color because it establishes the object histogram model based on the color information. So the effect of algorithm is not satisfactory when the color of object is similar to the background. Especially, algorithm requires that the tracked object shouldn't be occluded completely, which isn't consistent with the situation that the object may be occluded completely in the real world. An improved Camshift tracking algorithm based on the Kalman filtering was proposed by Wang and Yang [7] to solve the poor tracking ability problem in occlusions. The Kalman filtering used in object tracking is an optimal estimation method with the criterion of minimal error covariance. It has the advantages such as low calculation scale and high real-time performance. However, it supposes a linear Gauss state space model, which may be not consistent with the motion situation of the object in the real world. Considering this, an improved object tracking algorithm based on Camshift and the grey prediction model $\operatorname{GM}(1,1)$ [8] which has the advantages of sub-sample and poor information and doesn't assume the law of motion in advance is proposed to solve the poor tracking ability problem in occlusions in this paper. Experiment results of variant video sequences demonstrate that the proposed method can track the objects stably and accurately during occlusions.

\section{CAMSHIFT ALGORITHM}

The principle of Camshift is described as follows. Firstly, we convert the image into color probability distribution according to the color histogram of object. Secondly, we initialize the size and the position of the search window. Thirdly, the window is adjusted with the obtained result from previous frame adaptively. Finally, we can locate the center of the object in the current image by the result of search.

\section{A. Color probability distribution}

Firstly, we calculate the color histogram of the object. The RGB color space has weakness in representing shading effects or rapid illumination changing. To solve this problem, the Camshift algorithm adopts the HSV [9] ( Hue Saturation Value) color space to describe the object. Only $\mathrm{H}$ channel in HSV model can express the color information, so we extract it to make histogram. For each frame, the chrominance histogram is calculated as the following: Suppose that the number of total pixels in the frame is $n$, the chrominance grade of the histogram is $m$, and the chrominance grade 
index corresponding to the $\mathrm{i}$-th pixel ${ }^{x_{i}}$ is $\mathrm{c}\left(x_{i}\right)_{i=1, \cdots, n}$, then the element value of histogram $\left\{q_{u}\right\}_{u=1, \cdots, m}$ of this frame is

$$
q_{u}=\sum_{i=1}^{n} \delta\left[c\left(x_{i}\right)-u\right], u=1,2, \cdots, m,
$$

where $\delta$ is unit impulse function. The formula of histogram normalization is as following

$$
\left\{p_{u}=\min \left(\frac{255}{\max (q)} q_{u}, 255\right)\right\}_{u=1, \cdots, m} .
$$

We can normalize the value range of the histogram corresponding to every chrominance grade to the interval of $[0,255]$ so that through back projection the value of each pixel in the image is related to the corresponding to the value of each chrominance grade in the histogram. Thus the probability distribution graph of object-color is obtained.

\section{B. Mean shift algorithm}

Mean shift algorithm is a non-parametric estimation method based on gradient of density function which is proposed by Fukunaga and applied to pattern recognition firstly. To locate the object, it uses iterative search to find the extreme value of the probability distribution. The Mean shift algorithm tracking process is as follows:

(1) Select a search window in the probability distribution. The pixel position in the searching window is point $(x, y)$ and $I(x, y)$ is pixel value of point $(x, y)$ in the image.

(2) Calculation of a zero moments $M_{00}$, the first moment $M_{01}$ of $X$, and the first moment $M_{10}$ of $Y_{\text {by the }}$ following formulas:

$$
M_{00}=\sum_{x} \sum_{y} I(x, y), \quad M_{01}=\sum_{x} \sum_{y} y I(x, y),
$$$$
M_{10}=\sum_{x} \sum_{y} x I(x, y) .
$$

(3) The calculation of the center of mass $\left(x_{c}, y_{c}\right)$ for the search window

$$
\left(x_{c}, y_{c}\right)=\left(M_{10} / M_{00}, M_{01} / M_{00}\right) \text {. }
$$

Reset the size of the window

$$
s=2 \sqrt{M_{00} / 256} .
$$

Now we can get the size of the search window from (5). Move the center of the window to the centroid. If the moving distance is larger than preset-threshold, recalculate the centroid of the adjusted search window by (4). Adjust the position and size of the window until moving distance is less than preset-threshold.

\section{Camshift algorithm}

Extending the mentioned Mean Shift to a continuous video sequence, we get CamShift algorithm. The principle is to make Mean Shift algorithm apply to all the frame of the video sequence, and then regard the center and the size of the window obtained from the previous frame as the initial value of the search window in the next frame. Iterate this process, and we can succeed in the target tracking. The concrete steps are as follows.

Step 1. Initialize the search window.

Step 2. Calculate the back projection to obtain the color probability distribu-tion of the search window.

Step 3. Run Mean Shift algorithm to get the size and position of the new search window.

Step 4. In the next frame of the video, re-initialize the size and position of the search window with value of step 3 , and then jump to step 2.

Step 5. Calculate the long axis, short axis and orientation of the object.

By calculating the second order moment using the following formulas:

$$
M_{20}=\sum_{x} \sum_{y} x^{2} I(x, y), M_{02}=\sum_{x} \sum_{y} y^{2} I(x, y), \text { and } M_{11}=\sum_{x} \sum_{y} x y I(x, y),
$$

we can obtain lengths of the long axis and short axis of object in the frame respectively as:

$$
L=\sqrt{\frac{(A+C)+\sqrt{B^{2}+(A-C)^{2}}}{2}} \text {, and } S=\sqrt{\frac{(A+C)-\sqrt{B^{2}+(A-C)^{2}}}{2}},
$$

where

$$
A=M_{20} / M_{00}-x_{c}^{2}, B=M_{11} / M_{00}-x_{c} y_{c} \text {, and } C={ }^{M_{02}} / M_{00}-y_{c}^{2} \text {. }
$$

At the same time, we can also obtaim the direction angle $\theta$ of the long axis of object is as follow:

$$
\theta=\frac{1}{2} \arctan \left(\frac{2\left(M_{11} / M_{00}-x_{c} y_{c}\right)}{\left(M_{20} / M_{00}-x_{c}^{2}\right)-\left(M_{02} / M_{00}-y_{c}{ }^{2}\right)}\right) .
$$

When tracking the given object using Camshift algorithm, we only need to calculate the color probability distribution of the pixel in a larger region than the current window. Because we need not calculate probability distribution of all the pixels in every frame image, it reduces the computation and saves the searching time.

\section{GM(1,1) MODEL}

The grey system theory invented by professor Deng Julong is a new theory on uncertain problems with a small sample size or little information. Its primary application fields include data handling, feature analyzing, model building and trend predicting with sub-samples and uncertainty as presupposition.

Suppose that $X^{(0)}=\left(x^{(0)}(1), x^{(0)}(2), \cdots, x^{(0)}(n)\right)$ is the original sequence and $X^{(1)}=\left(x^{(1)}(1), x^{(1)}(2), \cdots, x^{(1)}(n)\right)$ is the first order accumulated generating sequence. Then the equation

$$
x^{(0)}(k)+a z^{(1)}(k)=b
$$

is called the original form of $\operatorname{GM}(1,1)$, where

$$
z^{(1)}(k)=0.5 x^{(1)}(k)+0.5 x^{(1)}(k-1), x^{(1)}(k)=\sum_{i=1}^{k} x^{(0)}(i), k=2,3, \cdot \cdots n \text {. }
$$


If $\hat{a}=(a, b)^{T}$ is a sequence of parameters, and

$$
Y=\left[x^{0}(2) x^{0}(3) \cdots x^{0}(n)\right]^{T}, B=\left[\begin{array}{cccc}
-z^{(1)}(2) & -z^{(1)}(3) & \cdots & -z^{(1)}(n) \\
1 & 1 & \cdots & 1
\end{array}\right]^{T},
$$

then the least square estimate sequence of the equation (6) satifies $\hat{a}=\left(B^{T} B\right)^{-1} B^{T} Y$. Thus, we can obtain the value of parameters $a, b$.

It is easy to know that the winterization equation of the equation (6) is the following differential equation

$$
\frac{d x^{(1)}}{d t}+a x^{(1)}=b \text {. }
$$

Obviously, the discrete solution of equation (7) is

$\hat{x}^{(1)}(k)=\left[x^{(0)}(1)-\frac{b}{a}\right] e^{-a(k-1)}+\frac{b}{a}, k=1,2, \cdots, n$.

Thus, the time respond sequences of the equation (6) is $\hat{X}^{(1)}=\left(\hat{x}^{(1)}(1), \hat{x}^{(1)}(2), \cdots, \hat{x}^{(1)}(n)\right)$.

So, the prediction function is

$$
x^{(0)}(k)=\left(1-e^{a}\right)\left[x^{(0)}(1)-\frac{b}{a}\right] e^{-a(k-1)} \text {. }
$$

\section{CAMSHIFT ALGORITHM BASED ON GREY PREDICTION}

\section{A. Similarity function measurement}

Bhattacharyya coefficient is chosen to measure the similarity of object model $q=\left\{q_{u}\right\}_{u=1, \cdots, m}$ and candidate model $p(y)=\left\{P_{u}(y)\right\}_{u=1, \cdots, m}$, the Bhattacharyya coefficient is defined as

$$
\rho(y)=\rho[P(y), q]=\sum_{u=1}^{m} \sqrt{p_{u}\left(y_{0}\right) q_{u}} .
$$

\section{B. The criterion of occlusion}

The similarity of object model and candidate model can be described by the Bhattacharyya coefficient whose value will decrease rapidly when the object is under cover. So we can judge whether the occlusion takes place according to the changing degree of the Bhattacharyya coefficient.

Given a threshold $T_{h}$, the criterion of the occlusion is:

$$
f\left(y_{k}\right)= \begin{cases}1, \text { normal tracking, } & \text { if } \rho\left(y_{k}\right) \geq T_{h}, \\ 0, \text { under cover, } & \text { others. }\end{cases}
$$

According to the above criterion, the tracking system will use the prediction value based on (8) to replace the original itinerate center when $f\left(y_{k}\right)=0$, and then the algorithm still can track the object until the object break from the cover.

\section{EXPERIMENTAL RESULTS AND ANALYSIS}

To demonstrate the validity and robustness of the proposed approach, we carry out object tracking test in a number of video sequences by Matlab 7.1. The experimental results are as follows: Figure 3 shows the tracking result of Camshift algorithm and Figure 4 shows the tracking result of approach proposed in this paper.
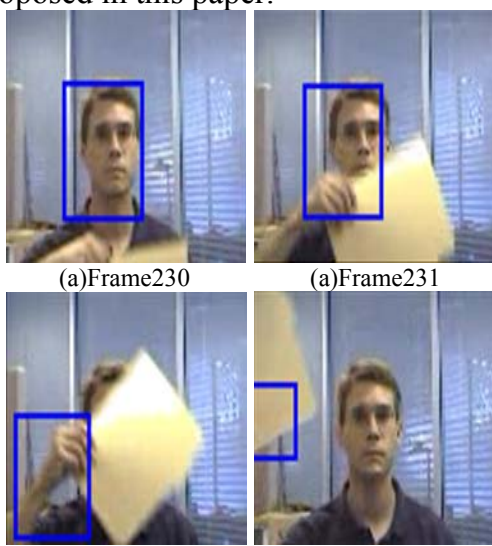

(a)Frame232

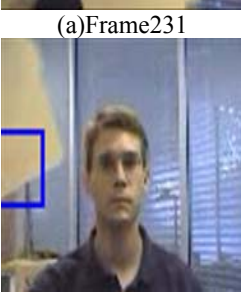

(a)Frame235

Figure 1. Traditional Camshift algorithm
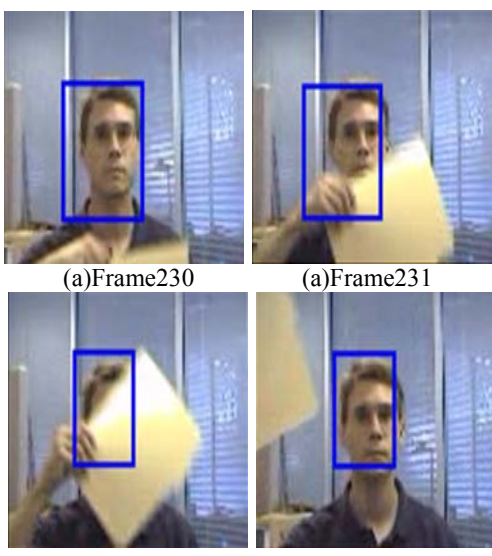

(a)Frame231

(a)Frame232

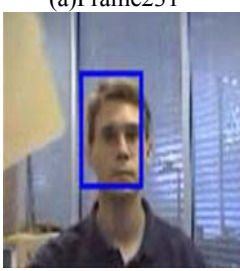

Figure 2. Result of this paper Method

From the tracking results shown in Fig. 1 and 2, it can be concluded that the proposed method can track the objects stably and accurately during occlusions.

\section{CONCLUSION}

Camshift algorithm has the advantage of better real-time, but the algorithm will track failure when the object is occlusioned seriously. An improved Camshift tracking algorithm based on the Kalman filtering was proposed to solve the problem of its poor tracking ability in occlusions. However, the Kalman filtering supposes a linear Gauss state space model, which may not be consistent with the motion situation of the object in the real world. Aiming to solve these problems, this paper presents an improved target tracking algorithm based on Camshift algorithm combined with GM (1.1) model. The results showed that the proposed algorithm can solve the above problems effectively and achieve real-time requirements. 


\section{ACKNOWLEDGMENT}

This work is supported by the NSFC (Grant Nos. 40801214) of China.

\section{REFERENCES}

[1] COMANICIU D; RAMESH V; MEER P. Real-time tracking of nongrid objects using mean shift. Proceedings of the IEEE Computer Society Conference on Computer Vision and Pattern Recognition[C], 2000, PP:142-149.

[2] FUKUNAGA K; HOSTETLER L D. The estimation of the gradient of a density function, with applications in pattern recognition, IEEE Transaction on Information Theory[J], 1975, PP:32-40

[3] CHENG Yi-zong. Mean shift, mode seeking, and clustering. IEEE Transaction Pattern Analysis and Machine Intelligence[J], 1995, PP:790-799.
[4] Comaniciu D; Meer P. Mean shift analysis and applications. IEEE Int. Conf. on Computer Vision[C], 1999, PP:1197-1203.

[5] BRADSKI R. Computer vision face tracking as a component of a perceptual user interface. Proceedings of IEEE Workshop Applications of Computer Vision[J]. 1998, PP:214-219.

[6] SUM Kai; LIU Shi-rong. Combined algorithm with modified Camshift and Kalman filter for multiobject tracking. Information and Control[J], 2009, PP:9-14.

[7] WANG Jiang-tao,YANG Jing-yu. Object Tracking Based on Kalman-Mean Shift in Occlusions. Journal of System Simulation[J], 2007, PP:4216-4220.

[8] LIU Si-feng; DANG Yao-guo; FANG Zhi-geng. Grey Theory and Applications. Beijing: Science Press[M], 2004, PP:126-133.

[9] Foley J D; Dam A V; Feiner S K; Hughes J F. Computer graphics: principles and practice in C, 2rd Edition, Addison-Wesley Professional[M], 1995, pp. 590-591 